\title{
ORIGINAL ARTICLE \\ Comparison of urodynamic findings in patients with upper and lower cervical spinal cord injury
}

\author{
S Sayılır, M Ersöz and S Yalçın
}

\begin{abstract}
Study design: Retrospective study.
Objectives: To analyze the neurogenic bladder characteristics and treatment approaches in patients with upper and lower cervical spinal cord injury (SCl) in order to make proper and reasonable decisions to the relevant patients.

Setting: Rehabilitation center in Ankara, Turkey.

Method: Ninety patients with cervical SCI were included. The urodynamic analyses of the patients were conducted retrospectively by using the urodynamic laboratory records. The patients were divided into two groups as the upper cervical SCI (UCSCI) group (C1-C5) and lower cervical SCl (LCSCl) group (C6-C8).

Results: In this study, 82 male (91.1\%) and 8 (8.9\%) female patients were included. There were 51 UCSCI patients with the mean age of $34.2 \pm 16.1$ years and $39 \mathrm{LCSCl}$ patients with the mean age of $30.4 \pm 12.5$ years. Detrusor overactivity and preservation of the bladder-filling sensation were significantly more frequent in the UCSCI group than in the $\operatorname{LCSCI}$ group $(P=0.048, P=0.000$ respectively). Moreover, there were statistically significant differences between the groups regarding the bladder-emptying methods, residual volume and the frequency of anticholinergic and alpha blocker use (all $P<0.05$ ). No significant difference was found between the groups regarding the frequency of autonomic dysreflexia, detrusor hypocompliancy and the bladder-storage and -emptying disorders (all $P>0.05$ ).
\end{abstract}

Conclusion: Our results demonstrate that there are differences in the upper and lower SCl cases in terms of neurogenic bladder characteristics and treatment approaches.

Spinal Cord (2013) 51, 780-783; doi:10.1038/sc.2013.83; published online 13 August 2013

Keywords: cervical spinal cord injury; urodynamics; neurogenic bladder

\section{INTRODUCTION}

Spinal cord injury (SCI) is a disease that is resulted by traumatic or nontraumatic reasons, and it causes restriction of self-care, mobilization and daily activities of the patients to a considerable degree. The SCI incidence was reported to be $15-40$ per million. ${ }^{1,2}$ The damage as a result of this injury not only limits the movement of the patient but also causes vital changes in the functions of many organs and lead to life-threatening complications. The aforementioned problems are more severe in cases of cervical SCI. Neurogenic bladder is commonplace in SCI patients. It decreases life span and adversely affects the quality of life. $^{3-6}$ Although the neurogenic bladder was one of the most important cause of morbidity/mortality in SCI patients in the past when the examination and treatment were not sufficiently developed, it has reached to a curable level today as a result of new developments.

The purpose of this study was to analyze the neurogenic bladder characteristics and treatment approaches retrospectively in the cervical SCI cases by using the urodynamic records of our hospital. As there were some differences in the functional abilities like hand function, sitting balance, and so on in upper (C1-C5) and lower (C6-C8) cervical SCI patients, we hypothesized that neurogenic bladder characteristics and treatment approaches would also differ in these groups. The aim of this study was to make proper and reasonable decisions for the neurogenic bladder treatment approaches in relevant patients with cervical SCI.

\section{MATERIALS AND METHODS}

Ninety patients with traumatic cervical SCI who had been referred to the urodynamics laboratory because of neurogenic bladder between 2002 and 2011 were included in this study. The age, gender, interval between the SCI and the urodynamic examination and etiological factor and SCI level according to American Spinal Injury Association Impairment Scale (AIS) classification of the patients were recorded. The patients were divided into two groups as the upper cervical SCI (UCSCI) group (C1-C5) and lower cervical SCI (LCSCI) group (C6-C8).

The urodynamic analyses of the patients were conducted retrospectively by using the urodynamic laboratory records. Urodynamic investigations were performed by the same investigator (ME) using a Libra + (MMS, Enschede, The Netherlands) urodynamic measurement system. Each patient was positioned a supine on a urological table. An 8-Fr 2-lumen catheter for adult patients and a 6-Fr 2-lumen catheter for pediatric patients were introduced transurethrally for bladder filling. The bladder was emptied. Continuous medium fill cystometry was performed at $50 \mathrm{ml} \mathrm{min}^{-1}$ for adults at $20 \mathrm{ml} \mathrm{min}^{-1}$ for children with room temperature sterile saline. Blood pressure monitoring was carried out during the cystometric examinations, and signs or symptoms of autonomic dysreflexia were monitored. The patients were informed about the bladder-filling sensations (the first sensation of bladder filling, the first desire to void and a strong desire to void) before the investigation and were asked to report all sensations related to bladder filling, but not to report if they do not feel anything. ${ }^{7-9}$ Filling was terminated when the patient had a strong desire to void, when significant leakage occurred, when intravesical pressure reached $40 \mathrm{~cm} \mathrm{H}_{2} \mathrm{O}$, observation of autonomic dysreflexia $(\mathrm{AD})$ and blood pressure 
changes (systolic blood pressure $>140 \mathrm{~mm} \mathrm{Hg}$ or $20-40 \mathrm{~mm} \mathrm{Hg}$ increase in the systolic blood pressure in adults and $15 \mathrm{~mm} \mathrm{Hg}$ increase in the systolic blood pressure in children ${ }^{10}$ ). The detrusor type and compliance, bladder-filling sensation, expected bladder capacity for children, maximum cystometric capacity, volume of micturated urine and residual volume were analyzed. The presence of storage and emptying disorder were determined according to the results of parameters analyzed in the filling and voiding phases. Additionally, the prescribed medical treatments and emptying methods were recorded.

The type of detrusor was classified as overactive, normoactive and underactive, detrusor hypocompliance was classified as present or absent ${ }^{11}$ and bladder-filling sensation was classified as absent, partially preserved and preserved. ${ }^{9}$ The compliance was considered as normal when the detrusor pressure was $\geqslant 20 \mathrm{ml}$ per $\mathrm{cm} \mathrm{H}_{2} \mathrm{O}$ and it was considered as hypocompliance when the detrusor pressure was $<20 \mathrm{ml}$ per $\mathrm{cm} \mathrm{H}_{2} \mathrm{O}^{11}$ The expected bladder capacity was calculated according to the age formula in the children (for children $<2$ years old: $(2 \times$ age +2$) \times 28 \mathrm{ml}$ and for children $>2$ years old: $($ age $/ 2+6) \times 28 \mathrm{ml}){ }^{12}$ The lower limit of the normal bladder capacity was determined as $300 \mathrm{ml}$ for adult patients. ${ }^{13-16}$ The lower limit was determined as $300 \mathrm{ml}$ in the children who were $\geqslant 10$ years old, similar to adult patients, as the expected bladder capacity measured with the formula was $>300 \mathrm{ml}$ in these children. Storage disorder presence was accepted if the maximum cystometric capacity measured during the filling phase was $<300 \mathrm{ml}$ in adult patients and in children $>10$ years and if the maximum cystometric capacity measured during the filling phase was lower than the expected bladder capacity in children $<10$ years. Besides, it was accepted that there was emptying disorder when the residual volume was $\geqslant 50 \mathrm{ml}$ in the adult patients and $\geqslant 20 \mathrm{ml}$ in the pediatric patients at the end of the voiding phase. The prescribed medical treatment (anticholinergic, alpha blocker and baclofen) and the prescribed emptying method (spontaneous voiding, emptying without catheter, intermittent catheterization and indwelling catheter) were recorded. The frequencies of self-intermittent catheterization and intermittent catheterization performed by the caregiver were also recorded.

SPSS software Version 15.0 (SPSS Inc., Chicago, IL, USA) was used in the evaluation of the data and $P<0.05$ was considered as statistically significant. $T$-test for independent samples was used in the comparison of

Table 1 Demographic and clinical features of the patients

\begin{tabular}{|c|c|c|c|}
\hline & $\begin{array}{l}\text { Upper cervical SCl } \\
(\text { C1-C5) }(\mathrm{n}=51)\end{array}$ & $\begin{array}{l}\text { Lower cervical SCl } \\
(\text { C6-C8) }(n=39)\end{array}$ & P-value \\
\hline Age (years) & $34.15 \pm 16.14$ & $30.41 \pm 12.52$ & \\
\hline Time (months) & $15.7 \pm 27.28$ & $5.76 \pm 6.57$ & \\
\hline Gender, n (\%) & & & 0.218 \\
\hline Male & $48(94.1)$ & $34(87.2)$ & \\
\hline Female & $3(5.9)$ & $5(12.5)$ & \\
\hline Etiological factor, $n(\%)$ & & & 0.246 \\
\hline Vehicle accidents & $20(39.2)$ & $20(51.3)$ & \\
\hline Fall from height & $12(23.5)$ & $7(17.9)$ & \\
\hline Other & 19 (36.9) & $12(31.8)$ & \\
\hline \multicolumn{4}{|l|}{ AIS classification, $n(\%)$} \\
\hline$A / S-A$ & $19(37,3)$ & $31(79.5)$ & \\
\hline$A / S-B$ & $13(25.5)$ & $5(12.8)$ & \\
\hline$A I S-C$ & $8(15.7)$ & $2(5.1)$ & \\
\hline$A / S-D$ & $11(26.6)$ & $1(2.6)$ & \\
\hline$A / S-E$ & 0 & 0 & \\
\hline Completeness of injury, $n(\%)$ & & & 0.000 \\
\hline Complete (AIS-A) & $19(37.3)$ & $31(79.5)$ & \\
\hline Incomplete (AIS B-E) & $32(62.7)$ & $8(20.5)$ & \\
\hline
\end{tabular}

Abbreviations: AIS, American Spinal Injury Association Impairment Scale; SCI, spinal cord injury. descriptive statistics and measured parameters. Chi-square test or Fischer's exact test was used when appropriate in the comparison of categorical parameters.

\section{RESULTS}

Demographic and clinical features of the patients are shown in Table 1. The comparisons of the urodynamic findings and therapeutic approaches are given in Table 2. Detrusor overactivity and preservation of the bladder-filling sensation were significantly more frequent in the UCSCI group than in the LCSCI $(P=0.048, P=0.000$ respectively). Moreover, there were significant differences between the groups regarding bladder-emptying methods, residual volume and frequency of anticholinergic and alpha blocker use (all $P<0.05)$. The less number of the patients who were administered baclofen in our study population limited the comparison of the usage of this agent in the groups. No significant difference were found between the groups regarding the frequencies of $\mathrm{AD}$, detrusor hypocompliancy and bladder-storage and -emptying disorders (all $P>0.05$ ).

We conducted Chi-square test, Fischer's exact test and T-test for independent samples for comparisons of complete UCSCI and LCSCI patients and incomplete UCSCI and LCSCI patients. There was no statistically significant difference between the complete UCSCI $(n=19)$ and complete LCSCI $(n=31)$ patients with respect to residual volume and frequencies of $\mathrm{AD}$, detrusor hypocompliancy, detrusor overactivity, bladder-storage and -emptying disorders and bladderemptying methods $(P>0.05)$. There was no statistically significant difference between incomplete UCSCI $(n=32)$ and incomplete LCSCI

Table 2 Comparison of urodynamic findings and therapuetic approaches in patients with upper and lower cervical spinal cord injury

\begin{tabular}{|c|c|c|c|}
\hline & $\begin{array}{l}\text { Upper cervical SCl } \\
(\text { C1-C5) }(\mathrm{n}=51)\end{array}$ & $\begin{array}{l}\text { Lower cervical SCl } \\
(\text { (C6-C8) }(\mathrm{n}=39)\end{array}$ & P-value \\
\hline Bladder-filling sensation & & & 0.000 \\
\hline Absent & $8(15.7)$ & $25(64.1)$ & \\
\hline Partially preserved & $39(76.5)$ & $14(35.9)$ & \\
\hline Preserved & $4(7.8)$ & 0 & \\
\hline Detrusor overactivity frequency & $45(88.2)$ & $28(71.8)$ & 0.048 \\
\hline $\begin{array}{l}\text { Detrusor hypocompliance } \\
\text { frequency }\end{array}$ & $39(76.5)$ & 31 (79.5) & 0.733 \\
\hline Storage disorder frequency & $41(80.4)$ & $30(76.9)$ & 0.689 \\
\hline Emptying disorder frequency & $46(90.2)$ & $37(94.9)$ & 0.412 \\
\hline $\begin{array}{l}\text { Residual urine }(\mathrm{ml}) \\
\text { (mean } \pm \text { s.d.) }\end{array}$ & $199.7 \pm 27.5$ & $286.6 \pm 29.1$ & 0.036 \\
\hline $\begin{array}{l}\text { Anticholinergic drugs } \\
\text { (prescribed) }\end{array}$ & $43(84.3)$ & $39(100)$ & 0.010 \\
\hline $\begin{array}{l}\text { Alpha-blocker drugs } \\
\text { (prescribed) }\end{array}$ & $11(21.6)$ & $1(2.6)$ & 0.009 \\
\hline $\begin{array}{l}\text { Autonomic dysreflexia } \\
\text { frequency }\end{array}$ & $29(56.9)$ & $21(53.8)$ & 0.775 \\
\hline Prescribed emptying method & & & 0.038 \\
\hline $\begin{array}{l}\text { Spontaneous voiding- } \\
\text { emptying without catheter }\end{array}$ & $12(23.5)$ & $2(5.1)$ & \\
\hline Intermittent catheterization & $17(33.4)$ & $20(51.3)$ & \\
\hline Indwelling catheter & $22(43.1)$ & $17(43.6)$ & \\
\hline Intermittent catheterization & & & 0.373 \\
\hline Self & $1(5.2)$ & $3(15.0)$ & \\
\hline Caregiver & $18(94.8)$ & $17(75.0)$ & \\
\hline
\end{tabular}

Abbreviation: $\mathrm{SCl}$, spinal cord injury.

Values are given as $n(\%)$ unless otherwise stated. 
$(n=8)$ patients with respect to residual volume and frequencies of $\mathrm{AD}$, detrusor hypocompliancy, detrusor overactivity, bladder-storage and -emptying disorders and bladder-emptying methods $(P>0.05)$. Comparisons of bladder-filling sensation could not be conducted because of the very low expected counts in Chi-square test.

\section{DISCUSSION}

The urinary system is one of the most important organ system that is involved in the SCI. Therefore, the urodynamic findings of the patients with cervical SCI were evaluated, and results were also significant by dividing the patients into two groups as UCSCI and LCSCI according to the neurogenic lesion levels in our study. Cases in the UCSCI and LCSCI groups were classified as complete (AIS-A) and incomplete (AIS B-E). In the UCSCI group, incomplete injured patients were significantly more than complete injured patients in our study. This significant difference may be explained by the higher mortality rates in complete UCSCI patients than in incomplete UCSCI patients. ${ }^{17}$

There was statistically significant difference in favor of the UCSCI group regarding bladder-filling sensation. This was attributed to the higher incomplete injury rate in the UCSCI group. The presence of bladder-filling sensation may be useful in the timing of bladder emptying. ${ }^{9}$ As the loss of bladder-filling sensation is frequent in the LCSCI group, we feel that strict and close follow-up for the bladderemptying timing and volume is required in this patient group.

The frequency of detrusor overactivity was significantly high in the UCSCI group. It is difficult to explain the underlying mechanism. A possible explanation may be the significantly higher number of incomplete injured patients in the UCSCI group. Higher rate of spasticity and hypereflexia in the incomplete injury than in the complete injury has been reported previously. ${ }^{18}$ In the light of this phenomenon, it is possible to say that higher rate of hypereflexia in detrusor muscle in the patients with incomplete lesions in the UCSCI group may be the reason for this significant difference.

Statistically significant difference was determined between the two groups when the patients were evaluated in terms of bladder-emptying method. Additionally, there was statistically significant difference between the groups in favor of the UCSCI group when the patients were compared in terms of residual volume. In the scope of these statistical data, the UCSCI group, including higher number of incomplete injured patients than in the LCSCI group, was in better condition in terms of urinary-emptying function reserve due to the higher frequency of spontaneous voiding and emptying without catheter and lower residual urine volume than the LCSCI group. This finding may be useful while making decisions on medical treatment and clinical approaches for neurogenic bladder in cervical SCI patients.

When the patients were evaluated in terms of anticholinergic and alpha blocker usage, statistically significant difference was observed between the groups. Frequencies of spontaneous voiding and emptying without catheter were higher and mean residual volume was lower in the UCSCI group than in the LCSCI group. Thus, alpha blocker prescribed in order to support and enhance the preserved emptying function was more frequent in the UCSCI group. On the other hand, higher rate of complete injury in the patients in the LCSCI group led to severe storage and emptying dysfunction of bladder. It is crucial to improve the bladder capacity in these patients. The higher rate of anticholinergic use in the LCSCI group was probably due to the severity in decrease of both the urinary storage and emptying function reserve in our study. Baclofen, which has positive effect on bladderemptying function by relaxing the external sphincter, is commonly used in SCI patients. However, the small number of the patients who were administered baclofen in our study population limited the comparison of the usage of this agent in the groups.

Self or third-party intermittent catheterization rates were not different statistically in the groups, although higher rate of intermittent self-catheterization was observed in the LSCSI group. Cardenas et al. ${ }^{19}$ reported that self-clean aseptic catheterization rate is higher in LCSCI patients than in UCSCI patients. The small number of patients in the subgroups and the higher rate of incomplete patients in the UCSCI group may be the reasons why there was no statistically significant difference between the two groups.

There was no statistically significant difference between the groups regarding $\mathrm{AD}$ rate during the urodynamic evaluations. However, $\mathrm{AD}$ symptom findings were determined in $55.6 \%$ of the patients. This rate indicates that proper precautions must be taken for the relevant patients.

Absence of statistical significant difference between complete UCSCI and complete LCSCI patients and between incomplete UCSCI and incomplete LCSCI patients with rescpect to residual volüme, the frequencies of $\mathrm{AD}$, detrusor hypocompliancy, detrusor overactivity, bladder-storage and -emptying disorders and bladder-emptying methods gave the impression that the higher rate of incomplete patients in the UCSCI group may have had a major role in the statistically significant differences observed between UCSCI and LCSCI patients in general. However, patient numbers in the complete and incomplete UCSCI and LCSCI subgroups were small, and further studies with larger sample sizes are required. Nevertheless, statistically significant differences observed between UCSCI and LCSCI patients in general is a clinical reality and keeping this finding in mind may be helpful in daily practice.

The small patient numbers in the complete and incomplete UCSCI and LCSCI subgroups, absence of uniform sphincteric electromyographic monitoring and relatively high filling rates appeared as limitations of our study. In our study, continuous medium fill cystometry was performed at $50 \mathrm{ml} \mathrm{min}^{-1}$ for adults and at $20 \mathrm{ml} \mathrm{min}^{-1}$ for children. Although $50 \mathrm{ml} \mathrm{min}^{-1}$ is the upper limit for medium fill cystometry, it can provoke detrusor overactivity and $\mathrm{AD}$ in patients with cervical lesions, and the significances of the urodynamic findings may be unclear, so the results should be interpreted accordingly. As this is a retrospective study including a period of 10 years, presence of some methodological limitations could not be avoided. Despite these limitations, we feel that our study is informative about the neurogenic bladder characteristics and neurogenic bladder treatment approaches in patients with cervical SCI.

\section{CONCLUSION}

In conclusion, the findings of the study not only provide comprehensive information about the neurogenic bladder in patients with cervical SCI, but also show that there are differences in the upper and lower SCI cases in terms of neurogenic bladder characteristics and therapeutic approaches. Herein presenting our study, we would like to contribute to the clinical approaches for neurogenic bladder.

\section{DATA ARCHIVING}

There were no data to deposit.

\section{CONFLICT OF INTEREST}

The authors declare no conflict of interest. 
1 Sekhon LH, Fehlings MG. Epidemiology, demographics and pathophysiology of acute spinal cord injury. Spine 2001; 26 (24 Suppl.): S2-12.

2 Karacan I, Koyuncu H, Pekel Ö, Sümbüloğlu G, Kırnap M, Dursun H et al. Traumatic spinal cord injuries in Turkey: a nation-wide epidemiologic study. Spinal Cord 2000; 38: 697-701.

3 Lee YH, Creasey GH, Lim H, Song J, Song K, Kim J. Detrusor and blood pressure responses to dorsal penile nerve stimulation during hyperreflexic contraction of the bladder in patients with cervical cord injury. Arch Phys Med Rehabil 2003; 84: $136-140$.

4 Morita H, Sazawa A, Kanno T, Keyanagi T. Long term urinary prognosis of cervical cord injury patients. Paraplegia 1994; 32: 30-35.

5 Kaplan SA, Chancellor MB, Blaivas JG. Bladder and sphincter behavior on patients with spinal cord lesions. J Urol 1991; 146: 113-117.

6 Ando M. Neurogenic bladder in patients with cervical cord compression disorders. Nihon Hinyokika Gakkai Zasshi 1990; 81: 243-250.

7 Wyndaele JJ. The normal pattern of perception of bladder filling during cystometry studied in 38 young healthy volunteers. J Urol 1998; 160: 479-481.

8 Wyndaele JJ, Wachter SD. Cystometrical sensory data from a normal population: comparison of two groups of young healthy volunteers examined with 5 years interval. Eur Urol 2002; 42: 34-38.

9 Ersöz M, Akyüz M. Bladder filling sensation in patients with spinal cord injury and the potential for sensation-dependent bladder emptying. Spinal Cord 2004; 42: 110-116.

10 Consortium For Spinal Cord Medicine, Clinical Practice Guidelines, August 2006
11 Guidelines on Neurogenic Lower Urinary Tract Dysfunction. European Association of Urology 2012.

12 Ersöz M. Neurogenic bladder and bowel in pediatric spinal cord injury patients. Turk J Phys Med Rehab 2008; 2: 75-80.

13 Robertson AS, Griffiths CJ, Ramsden PD, Neal DE. Bladder function in healthy volunteers: ambulatory monitoring and conventional urodynamic studies. $\mathrm{Br} \mathrm{J} \mathrm{Urol}$ 1994; 73: 242-249.

14 Abdel-Rahman M, Coulombe A, Devroede G, Toppercer A, Duguay C, Lapointe L et al. Urorectodynamic evaluation of healthy volunteers. Urology 1982; 19: 559-564.

15 Dmochowski R. Cystometry. Urol Clin North Am 1996; 23: 243-252.

16 Ersöz M, Tunç H, Akyüz M, Özel S. Bladder storage and emptying disorder frequencies in hemorrhagic and ischemic stroke patients with bladder dysfunction. Cerebrovasc Dis 2005; 20: 395-399.

17 DeVivo MJ, Krause JS, Lammertse DP. Recent trends in mortality and causes of death among persons with spinal cord injury. Arch Phys Med Rehabil 1999; 80: 1411-1419.

18 Kirshblum S. Rehabilitation of SCl. In: DeLisa JA and Gans BM (eds) Physical Medicine and Rehabilitation, Principles and Practice, 4th edn. Lippincott Williams \& Wilkins: Philadelphia, PA, USA, 2005, pp 1715-1751.

19 Cardenas DD, Mayo ME. Management of bladder dysfunction. In: Braddom RL (ed.) Physical Medicine and Rehabilitation, 4th edn. Saunders Elsevier: Philadelphia, PA, USA, 2010, pp 626 